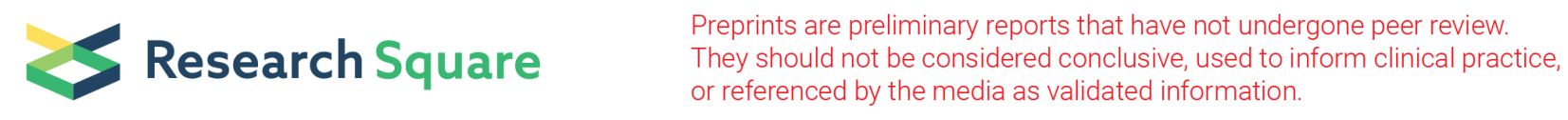

\title{
No Observed Risk of Pregnancy and Maternal-Fetal Transmission of Zika Virus Following Blood Transfusion
}

\author{
Valeria DeGiorgi \\ National Institutes of Health Clinical Center \\ Jennifer Tan \\ National Institutes of Health Clinical Center \\ Sarah Fowler \\ National Institutes of Health Clinical Center \\ Sonia Maciejewski \\ National Institute of Allergy and Infectious Diseases

\section{David Gordon} \\ National Institute of Allergy and Infectious Diseases \\ Harvey J. Alter \\ National Institutes of Health Clinical Center \\ Robert D Allison ( $\nabla$ traveldoc365@gmail.com ) \\ National Institutes of Health https://orcid.org/0000-0001-8458-5250
}

\section{Research article}

Keywords: Zika Virus, Blood Transfusion, Transfusion-Transmitted Infection, Fetomaternal Infection Transmission, Fetomaternal, Infection Transmission, Maternal-Fetal, Infection Transmission, Maternal-Fetal Infection Transmission, Mother-to-Child Transmission

Posted Date: August 6th, 2019

DOI: https://doi.org/10.21203/rs.2.12432/v1

License: () This work is licensed under a Creative Commons Attribution 4.0 International License. Read Full License 


\section{Abstract}

BACKGROUND: Zika virus (ZIKV) is primarily mosquito-borne, but can also be transfusion- and sexually transmitted. Maternal-fetal transmission may result in fetal loss or severe congenital defects. METHODS: We surveyed blood recipients enrolled in a donor-recipient-linked study of transfusiontransmitted infections to assess potential risk of ZIKV maternal-fetal transmission following transfusion. Participants were tested for antibodies to ZIKV, dengue and chikungunya viruses. Positive serology from post- and pre-transfusion samples and from linked donors were tested using real-time PCR. RESULTS: The mean age of female recipients was 65 years (36-80). Only one was $<50$ years. None reported having sexual intercourse within the first month and none became pregnant within six months following transfusion. Male recipients were a mean 62 years of age (31-81). Twenty percent of males had sexual intercourse with a female partner within three months of transfusion; no pregnancies resulted. Females and males most commonly reported that they were no longer sexually active or felt too ill after transfusion. All of the respondents were IgM and RT-PCR negative. CONCLUSIONS: Our survey of blood recipients, combined with derived data and the literature, suggest that most transfused women are beyond the age of likely pregnancy and most transfused women and men are too infirm from the underlying condition to engage in sex. However, $20 \%$ of male recipients did report having sexual intercourse during the ZIKV infectious period.

\section{Background}

Zika virus (ZIKV) is primarily mosquito-borne, but can also be transfusion- and sexually transmitted. ${ }^{1-3}$ Maternal-fetal transmission may result in fetal loss or severe congenital defects. ${ }^{4-6}$ Transfusion-transmission of ZIKV to a pregnant blood recipient with subsequent vertical transmission and birth defects are the most feared consequences of transfusion-related ZIKV. Following

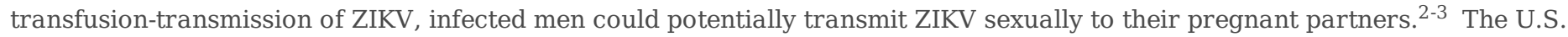
Food and Drug Administration (FDA) issued guidance in 2016 that required individual donor nucleic acid testing (ID-NAT) of all U.S. blood donors for ZIKV. ${ }^{7}$ An FDA revision was issued in 2018 that allows donor nucleic acid testing in mini-pools (MP-NAT) with conversion to ID-NAT if pre-defined thresholds are met (e.g. local or regional ZIKV-positive donor). ${ }^{8}$ Both strategies, ID-NAT and MP-NAT, come with considerable cost. ${ }^{9-12}$

We surveyed blood recipients enrolled in a donor-recipient-linked prospective study of transfusion-transmitted infections, performed ZIKV, DENV and CHIKV serologic and RT-PCR testing and collected demographic and clinical laboratory data from the time of transfusion. The main aims of the study were to assess the likelihood of pregnancy or sexual transmission to a pregnant woman following transfusion, and by extension, the likelihood that transfusion-transmitted ZIKV could result in vertical transmission.

\section{Methods}

\section{Zika virus exposure and pregnancy risk surveys}

Blood transfusion recipients were surveyed who enrolled in the donor-recipient-linked prospective study, Transfusion-Related Infections Prospectively Studied (TRIPS; NCT00023023) from the beginning of the ZIKV epidemic period (November 2015) until routine blood donor screening for ZIKV RNA was implemented in the U.S. (December 2016). Surveys were administered by telephone to assess the likelihood of pregnancy among participants transfused during the ZIKV epidemic period and to assess by extension, the risk of ZIKV transmission to a fetus following a ZIKV transfusion-transmitted infection. Recipients were called at least three times, at different times of the day and on separate days over a 4-week period. Recipients whose phone numbers had changed, or who didn't return our calls were sent a letter requesting they contact us. When all efforts to contact them failed during a 4-week period, they were considered lost to follow-up. All blood recipients who completed the survey provided verbal informed consent after an IRB-approved script was read to them.

Female transfusion recipients were asked the following questions: did you have sexual relations during the first month following your blood transfusion? If so, did you become pregnant? If you became pregnant, what was the outcome of the pregnancy? If you became pregnant, are you aware of any birth defects? Male transfusion recipients were asked the following questions: did you have sexual relations during the 3 months following your blood transfusion? If so, was your partner pregnant, or 
did your partner become pregnant? If your partner became pregnant, what was the outcome of the pregnancy? If your partner became pregnant, are you aware of any birth defects? Male recipients were asked about sexual history 3 months post-transfusion because nearly all ZIKV RNA-positive semen samples are detected within this period. ${ }^{13}$ Semen containing ZIKV is likely only infectious within the first 30 days of infection. ${ }^{13}$ Both female and male recipients were then asked if they had any additional comments or questions.

\section{Study population and specimens}

The TRIPS study was initiated in 2001 and includes linked donor-recipient peripheral blood specimens from transfusion recipients who were enrolled in the National Institutes of Health Clinical Center (NIH) or from Johns Hopkins Suburban Hospital (JHSH), both located in Bethesda, Maryland, USA. All samples were collected from patients whose indication for transfusion was related to chemo- or immunotherapy (NIH), oncologic surgery (NIH) or cardiac surgery (JHSH). Plasma samples from blood product recipients were collected pre-transfusion, and then at 4, 8, 12 and 24 weeks post-transfusion. Donor and recipient samples were stored in 1-2 $\mathrm{mL}$ aliquots at $-80^{\circ} \mathrm{C}$ in a central repository (SeraCare BioServices, Gaithersburg, MD). Medical records of recipients were reviewed to collect demographics and detailed information about the transfusion including indication(s), types and volume of products and co-morbid medical conditions and treatments. Patients were eligible to enroll in the TRIPS study if they were 18 years of age or older, received a blood transfusion at NIH or JHSH and resided in the U.S. during the six-month follow-up period. Transfusion recipients were excluded from the study if they had a poor prognosis with a life expectancy of less than three

months and/or had been transfused within the past 6 weeks. For the latter, we assumed that an infection caused by a transfusion $>6$ weeks prior to enrollment would show up in the pre-sample. An infection caused by a transfusion within 6 weeks of enrollment might still be in an incubation period at enrollment and confound the analysis of a subsequent (within study) viremia or antibody seroconversion.

All study participants provided informed consent prior to sample collection. The study was approved and is reviewed annually by NIH and Johns Hopkins institutional review boards (IRB).

\section{Laboratory Testing}

Detailed descriptions of all laboratory testing methodology can be found in Appendix A. Briefly, clinical laboratory testing was performed on samples collected within 24 hours before transfusion by the respective departments of laboratory medicine in the NIH Clinical Center or JHSH. Flow cytometry was performed by the NIH Clinical Center Department of Laboratory Medicine on immunosuppressed blood recipients within 1 week before transfusion.

Recipient plasma was tested by ELISA for ZIKV, DENV and CHIKV-specific IgM and IgG at 4 weeks post-transfusion. DENV and CHIKV were tested because they share the same Aedes agypti vector mosquito with ZIKV and may exist as a co-infection. ${ }^{14}$ Cases of DENV transmission via blood transfusion have been documented, and CHIKV has the potential to be transmitted via this route. ${ }^{15,16}$ Further, there is evidence for DENV-related fetal congenital defects, though sexual transmission has not been documented. ${ }^{17}$ Recipients with positive post-transfusion serology results had their pre-transfusion samples tested for virus-specific IgM and IgG by ELISA and for viral RNA by real-time PCR.

\section{Results}

\section{Study Cohort}


Of 856 immunosuppressed or high-volume blood recipients who received at least one transfusion at NIH or JHSH during the ZIKV epidemic period, 260 (30\%) met the TRIPS study inclusion and/or exclusion criteria and were enrolled. Among the remaining 596 cases, 278 (47\%) were excluded for a history of transfusion within 6 weeks, 201 (34\%) for residing outside of the U.S. during the six month follow-up period, 68 (11\%) for having a poor prognosis and life expectancy less than three months, and 49 (8\%) for being younger than 18 years old.

Sixty-four (25\%) of the two hundred sixty enrolled TRIPS study participants had samples available for ZIKV, DENV and CHIKV testing, and 46 (18\%) agreed to participate in this survey-based sub-study.

\section{Characteristics and Responses from Surveyed Blood Recipients}

Characteristics of surveyed blood recipients are given in Table 1 . The average age at transfusion was $63 \pm 12$ years, 16 (35\%) were female and 29 (63\%) were Caucasian. Among all recipients, the mean hemoglobin was 10.7 g/dL (normal range 11.215.7), mean hematocrit was 31.7\% (34.1-44.9) and mean platelet count was $179 \mathrm{~K} / \mathrm{uL}$ (173-369). On average, recipients received 5.9 units of blood products. Four (9\%) recipients had non-surgical indications for blood transfusion, primarily for cancer-related cytopenias, and 42 (91\%) recipients had a surgical indication, primarily cardiac. Cardiac surgeries included 23 coronary artery bypass graft cases, 3 aortic valve repairs, 1 abdominal aortic aneurysm repair, 1 mitral valve repair, 14 partial nephrectomies and 1 radical cystectomy. Five cardiac patients had more than 1 procedure during the same surgery; none had more than 2 procedures. Compared to non-immunosuppressed recipients, immunosuppressed recipients had significantly lower mean white blood cell counts 3.5 vs. $8.5 \mathrm{~K} / \mathrm{uL}$; $\mathrm{P}=0.003$ ) and absolute neutrophil counts (3.0 vs. $6.8 \mathrm{~K} / \mathrm{uL}$; $\mathrm{P}=0.021)$.

Forty-six (72\%) of 64 enrolled recipients were successfully contacted by telephone and agreed to complete the survey. Eleven (17\%) had died, 6 (9\%) were lost to follow-up and 1 (2\%) declined to participate. The mean age of survey respondents was $63 \pm 12$ years, 16 (35\%) were female, and 29 (63\%) were Caucasian. The demographics of survey respondents were similar to all enrolled blood recipients (data not shown).

Among females, the average age was $65 \pm 12$ years (range, 36-80); only one female recipient was less than 50 years old. None of the female respondents reported having sexual intercourse within the first month following transfusion and none became pregnant within 6 months post-transfusion. After answering the survey questions, 14 (88\%) of female respondents offered an elaboration of their survey answers: 7 (50\%) of the woman said they had not been sexually active for many years; 4 (29\%) said they felt too ill to have sexual relations for months after transfusion; and 3 (21\%) of the women noted that they were still in the hospital 1 month after transfusion.

Among males, the average age was $62 \pm 12$ years (range, 31-81). Six (20\%) of the total 30 male respondents reported having sexual intercourse with a female partner during the 3 months following transfusion. Sexually active males reported that no pregnancies resulted from any sexual encounter during this time period. Eleven of twenty-four (46\%) male respondents who did not have sex after transfusion volunteered additional information: 4 (36\%) said they were no longer sexually active; 3 (27\%) said they suffered from impotence; 3 (27\%) felt too ill to have sexual relations 3 months following transfusion; 1 man (9\%) was still in the hospital 3 months after transfusion.

\section{Serologic, FRNT and Molecular Testing}

Serum specimens from 46 surveyed transfusion recipients and 19 linked donors were tested for the presence of IgM and IgG antibodies to ZIKV, DENV and CHIKV by ELISA. None of the recipients or their donors had detectable ZIKV or DENV IgM. CHIKV IgM was detectable in one blood recipient, however the sample was focus reduction neutralization test (FRNT) and CHIKV RNAnegative, and the donor-linked samples were CHIKV IgM, IgG and RNA-negative, supporting a false-positive test result. Virusspecific IgG antibodies were detectable for ZIKV in 3 recipients, for DENV in 6 recipients and for CHIKV in 2 recipients; all were 
male. These IgG-positive recipient specimens were all RNA negative. However, in five of these cases, both donors and a linkedrecipient were positive for IgG, suggesting passive antibody transfer through transfusion: two recipients and a linked donor were both ZIKV and DENV IgG positive; and an additional three recipients and a linked donor were both DENV IgG positive.

\section{Discussion}

The risk of exposure to blood products during pregnancy, and therefore the risk of ZIKV transfusion-transmission to a pregnant woman, appears to be very low. Murphy et. al. analyzed over forty-five thousand pregnancies in a large tertiary care center and found that only $0.124 \%$ of expectant mothers received a transfusion at some point in their pregnancy. ${ }^{18} \mathrm{~A}$ less recent study using U.S. hospital discharge data reported a range of 0.24 to $0.46 \%{ }^{19}$ ZIKV is thought to be most efficiently transmitted during the first trimester, and in Murphy's study only $0.04 \%$ received a transfusion during this period. ${ }^{6,18}$

The results of our blood recipient survey suggest that if a female blood recipient was infected with ZIKV, the likelihood of pregnancy following transfusion, and by extension transmission to a fetus, is very low. The average age of our female blood recipients was 65 years, which is similar to a large study of U.S. blood recipient demographics. ${ }^{20,21}$ None of our female respondents had sexual intercourse within 4 weeks of transfusion; ZIKV is typically no longer detectable in blood 14 days after infection. ${ }^{22}$ About half had not been sexually active for many years and the other half felt too ill to have sex, or were still in the hospital 4 weeks after transfusion.

Our results also suggest that if a male blood recipient was infected with ZIKV within 3 months of transfusion, the probability of sexual transmission to a female pregnant partner, or sexual intercourse that results in pregnancy is likely low. However it is possible: twenty percent of male recipients reported having sexual intercourse within the Zika virus infectious period;

Taken together, the risk of maternal-fetal transmission of ZIKV related to transfusion is likely low and the cost of universal blood donor testing is substantial. In August 2016, FDA recommended screening all donations for ZIKV by ID-NAT. ${ }^{7}$ With nearly 14 million blood donors per year in the 50 states and Washington, D.C., and assuming \$10 per individual donor nucleic acid test (IDNAT), Ellingston et al. estimated a cost of \$137 million per year for universal ZIKV donor screening using ID-NAT. ${ }^{10}$ After screening over 4 million donations using transcription-mediated amplification (ID-NAT), Saá et al. calculated a cost of \$5.3 million per ZIKV-positive infection. ${ }^{11}$ In July 2018, FDA issued revised guidance recommending routine screening of all donors for ZIKV using ID-NAT or MP-NAT. ${ }^{8}$ The yearly cost of universal MP-NAT testing was estimated as $\$ 82$ million per year. ${ }^{10}$ In a study by Russell et. al., MP-NAT was cost-effective in Puerto Rico only during mosquito season, but no screening policy was cost-effective in the 50 states. $^{12}$

None of the forty-six survey respondents had a transfusion-transmitted ZIKV, DENV or CHIKV infection. The small sample size limits our ability to make any generalization about risk of transfusion-transmission of these agents. However, two recipient and linked-donor pairs were ZIKV and DENV IgG-positive and an additional three were DENV IgG-positive alone. This suggests passive antibody transfer, prior infection in the donor, and the potential for transfusion-transmission of these viruses. Our study population includes surgical patients, particularly cardiac and immunosuppressed patients, who may be less likely to have sexual intercourse after transfusion compared to other recipient populations. However, our blood recipients may also be at greater risk for transfusion-transmitted infections than typical recipients.

\section{Conclusion}


Although this study is too small to draw broad inferences, the derived data and the literature suggest that many, and probably most, woman who are transfused are beyond the age of likely pregnancy and by extension, of maternal-fetal ZIKV transmission and associated birth defects. Further, most transfused women and men are too infirm from the underlying condition that necessitated transfusion to engage in sex during the interval when the ZIKV virus circulates in the blood stream or is most likely to be present in semen. Twenty percent of male recipients did report having sexual intercourse during the ZIKV infectious period. Though these circumstances make it unlikely that a blood transfusion would secondarily result in a ZIKV-related birth defect, they do not exclude the possibility.

\section{References}

1. Kuehnert MJ, Basavaraju SV, Moseley RR et al. Screening of Blood Donations for Zika Virus Infection - Puerto Rico, April 3-June 11, 2016. MMWR. Morbidity and mortality weekly report. 2016; 65(24):627-628.

2. D'Ortenzio E, Matheron S, Yazdanpanah Y et al. Evidence of Sexual Transmission of Zika Virus. N Engl J Med. 2016 Jun 2 ; 374 (22):2195-8.

3. Musso D, Roche C, Robin et al. Potential sexual transmission of Zika virus. Emerg Infect Dis. 2015 Feb; 21(2):359-61.

4. Besnard M, Lastere S, Teissier A et al. Evidence of perinatal transmission of Zika virus, French Polynesia, December 2013 and February 2014. Euro Surveill. 2014; 19.

5. Coffey LL, Keesler RI, Pesavento PA et al. Intraamniotic Zika virus inoculation of pregnant rhesus macaques produces fetal neurologic disease. Nat Commun. 2018 Jun 20;9(1):2414.

6. Johansson MA, Mier-y-Teran-Romero L, Reefhuis et al. Zika and the Risk of Microcephaly. N Engl J Med. 2016 Jul 7; 375 (1):1-4.

7. Food and Drug Administration. Revised recommendations for reducing the risk of Zika virus transmission by blood and blood components. 2016

(https://www.fda.gov/gov/biologicsbloodvaccines/guidancecomplianceregulatoryinformation/guidances/blood/ucm518213.pdf).

8. Food and Drug Administration. Revised recommendations for reducing the risk of Zika virus transmission by blood and blood components. 2018

(https://www.fda.gov/gov/biologicsbloodvaccines/guidancecomplianceregulatoryinformation/guidances/blood/ucm518213.pdf).

9. Bloch EM, Ness PM, Tobian AAR, Sugarman J. Revisiting Blood Safety Practices Given Emerging Data about Zika Virus. N Engl J Med. 2018 May 10; 378 (19):1837-1841.

10. Ellingson KD, Sapiano MRP, Haass KA et al. Cost projections for implementation of safety interventions to prevent transfusiontransmitted Zika virus infection in the United States. Transfusion. 2017 Jun; 57 Suppl 2:1625-1633. 
11. Saá P, Proctor M, Foster G, Krysztof D, Winton C et al. Investigational Testing for Zika Virus among U.S. Blood Donors. N Engl J Med. 2018 May 10;378 (19):1778-1788.

12. Russell WA, Stramer SL, Busch MP, Custer B. Screening the blood supply for Zika virus in the 50 U.S. states and Puerto Rico: A cost-effectiveness analysis. Ann Intern Med. 2019 Jan 8 [Epub ahead of print]

13. Mead PS, Duggal NK, Hook SA, Delorey M, Fischer M et al. Zika Virus Shedding in Semen of Symptomatic Infected Men. N Engl J Med. 2018 Apr 12; 378 (15):1377-1385.

14. Carrillo-Hernández MY, Ruiz-Saenz J, Villamizar LJ, Gómez-Rangel SY et al. Co-circulation and simultaneous co-infection of dengue, chikungunya, and zika viruses in patients with febrile syndrome at the Colombian-Venezuelan border. BMC Infect Dis. 2018 Jan 30; 18 (1):61.

15. Chen LH, Wilson ME. Update on non-vector transmission of dengue: relevant studies with Zika and other flaviviruses. Trop Dis Travel Med Vaccines. 2016 Aug 29; 2:15.

16. Slavov SN, Otaguiri KK, Bianquini ML et al. Seroprevalence of chikungunya virus in blood donors from Northern and Southeastern Brazil. Hematol Transfus Cell Ther. 2018 Oct-Dec;40(4):358-362.

17. Paixão ES, Teixeira MG, Costa MDCN, Barreto ML, Rodrigues LC. Symptomatic Dengue during Pregnancy and Congenital Neurologic Malformations. Emerg Infect Dis. 2018 Sep;24(9):1748-1750.

18. Murphy MS, Shehata N, Colas JA et al. Risk of exposure to blood products during pregnancy: guidance for Zika and other donor deferral policies. Transfusion. 2017 Mar; 57:811-815.

19. Kuklina EV, Meikle SF, Jamieson DJ et al. Severe obstetric morbidity in the United States: 1998-2005. Obstet Gynecol. 2009 Feb; 113:293-9.

20. Edgren G, Murphy EL, Brambilla DJ, Westlake M, Rostgaard K, Lee C, Cable RG,Triulzi D, Bruhn R, St Lezin EM, Erikstrup C, Ullum H, Glynn SA, Kleinman S, Hjalgrim H, Roubinian NH; NHLBI Recipient Epidemiology and Donor Evaluation Study-III (REDS-III) Group. Association of Blood Donor Sex and Prior Pregnancy With Mortality Among Red Blood Cell Transfusion Recipients. JAMA. 2019 Jun 11;321(22):2183-2192.

21. Caram-Deelder C, Kreuger AL, Evers D, de Vooght KMK, van de Kerkhof D, Visser O, Péquériaux NCV, Hudig F, Zwaginga JJ, van der Bom JG, Middelburg RA. Association of Blood Transfusion From Female Donors With and Without a History of Pregnancy With Mortality Among Male and Female Transfusion Recipients. JAMA. 2017 Oct 17;318(15):1471-1478.

22. Paz-Bailey G, Rosenberg ES, Doyle Ket al. Persistence of Zika Virus in Body Fluids - Final Report. N Engl J Med. 2017 Feb 14; 379 (13):1234-1243.

\section{Tables}

Table 1. Characteristics of Blood Recipients 


Yes No

No. (\%)

Age at transfusion, mean yrs (+/- SD)

Females, No. (\%)

Caucasian, No. (\%)
$46(100)$

$63(12)$

16 (35)

29 (63)
4 (9)

$58(10)$

1 (2)

4 (9)
42 (91)

$63(12)$

15 (33)

$25(54)$
P-Value

NA

0.352

0.667

0.632

\begin{tabular}{|c|c|c|c|c|}
\hline \multicolumn{5}{|l|}{ Laboratory data, mean (+/- SD) } \\
\hline HGB (11.2-15.7 g/dL) & $10.7(2.6)$ & $8.4(1.5)$ & $11.0(2.6)$ & 0.056 \\
\hline HCT $\quad(34.1-44.9 \%)$ & $31.7(7.4)$ & $24.4(3.5)$ & $32.4(7.3)$ & 0.037 \\
\hline PLT $(173-369 \mathrm{~K} / \mathrm{uL})$ & $179(69)$ & $166(170)$ & $180(54)$ & 0.688 \\
\hline WBC $(3.98-10.04 \mathrm{~K} / \mathrm{uL})$ & $8.1(3.4)$ & $3.5(4.0)$ & $8.5(3.0)$ & 0.003 \\
\hline ANC $(1.56-6.13 \mathrm{~K} / \mathrm{uL})$ & $6.3(3.1)$ & $3.0(3.2)$ & $6.8(2.8)$ & 0.021 \\
\hline \multicolumn{5}{|l|}{ Transfused units per recipient, mean (+/- SD) } \\
\hline Total & $5.9(4.2)$ & $2.8(1.5)$ & $6.2(4.3)$ & 0.115 \\
\hline Red blood cells & $3.2(2.7)$ & $2.5(1.0)$ & $3.3(2.8)$ & 0.573 \\
\hline Platelets & $1.1(1.4)$ & $0(0)$ & $1.2(1.5)$ & 0.021 \\
\hline Fresh frozen plasma & $1.6(2.1)$ & $0.25(0.5)$ & $1.7(2.1)$ & 0.187 \\
\hline \multicolumn{5}{|l|}{ Indications for transfusion, No. (\%) } \\
\hline Non-surgical (cancer-related cytopenias) & $4(9)$ & $4(9)$ & $0(0)$ & \multirow[t]{2}{*}{$<0.001$} \\
\hline Surgical & $42(91)$ & $0(0)$ & $42(91)$ & \\
\hline Non-cardiac & $14(33)$ & $0(0)$ & $14(33)$ & \multirow[t]{2}{*}{ NA } \\
\hline Cardiac* & $28(67)$ & $0(0)$ & $28(67)$ & \\
\hline
\end{tabular}

SD, standard deviation; HgB hemoglobin (normal range, 11.2-15.7 g/dL); HCT, hematocrit (34.1-44.9 \%); PLT, platelet count (173-369 K/uL); WBC, while blood cell count (3.98-10.04 K/uL); ANC, absolute neutrophil count (1.56-6.13 K/uL); CD4, CD4 T-cell count (359-1565/uL); CD8, CD8 T-cell count (178-853/uL); CD19, CD19 B-cell count (61-321/uL); NK, natural killer cell count (126-729/uL); NA, not applicable

\section{Abbreviations}

Zika virus, ZIKV; U.S. Food and Drug Administration, FDA; individual blood donor nucleic acid testing, ID-NAT; blood donor nucleic acid testing in mini-pools, MP-NAT; dengue virus, DENV; chikungunya virus, CHIKV; National Institutes of Health Clinical Center, NIH CC; Johns Hopkins Suburban Hospital, JHSH; focus reduction neutralization test, FRNT; Transfusion-Related Infections Prospectively Studied, TRIPS.

\section{Declarations}

\section{Ethics approval}

All study participants provided informed consent prior to sample collection. The study was approved and is reviewed annually by the National Heart Lung and Blood Institute of the National Institutes of Health and the Johns Hopkins Medicine institutional review boards.

Availability of data and materials

The datasets used and/or analyzed during the current study are available from the corresponding author on reasonable request.

Funding

This research was supported by the National Institutes of Health (NIH) Clinical Center, part of the NIH Intramural Research Program. 
The authors declare they have no competing interests.

Authors' contributions

VDG developed and led testing protocols. VDG and JT performed and analyzed ELISA and RT-PCR testing and made major contributions to methods. SF enrolled and coordinated human subjects' visits, clinical data and sample collection. SM and DG performed and analyzed FRNT and made major contributions to methods. RDA conceived, designed and led the study, analyzed and interpreted data. VDG, JT, HJA and RDA made major contributions to writing the manuscript. All authors read and approved the final manuscript.

Acknowledgments

We thank the participants of our transfusion-related infections prospective study and Johns Hopkins Suburban Hospital staff, including Philip Maglo and Jackie Lobien, for screening and enrolling cardiac surgery patients. We also thank Melissa Puig Lluch who was an NIH research assistant during the study.

\section{Supplementary Files}

This is a list of supplementary files associated with this preprint. Click to download.

- supplement1.docx 